\title{
Simulation Research on Redundant Mechanism of Dual CAN Bus
}

\author{
Lilong Tan, Le Yang, Shuai Li, Li Zhang \\ The Department of Mechanical and Electrical Control, Xi'an Research Institute of Hi-tech, Xi'an \\ 710025, P.R.China \\ 617816007@qq.com
}

Keywords: DSP, CAN bus, redundant message, reliability

\begin{abstract}
In order to improve the reliability of redundant CAN bus communication network, in this paper, a dual CAN bus redundancy communication system based on DSP is designed. In the hardware, a dual CAN redundant interface circuit based on TMS320F2808 chip is designed; in the software, a dual CAN bus communication scheme is proposed, system initialization procedure and redundant message filtering mechanism is designed. The test result indicated that the system can realize the function of transmitting data message at the same time, and when there was something wrong with one bus, another bus can continue to transmit data, the stability and reliability of communication system is improved.
\end{abstract}

\section{Introduction}

In recent years, with the rapid development of information technology and the automation level of industrial production, the number of nodes in CAN network is more and more. But the incidence of node and CAN bus failures in the network is also more and more at the same time [1]. The reliability of CAN network is very important especially in the occasion of high reliability requirement of weapon system. So, how to improve the reliability of CAN bus system is the key direction of research at home and abroad in recent years.

At present, the methods of improving the reliability of CAN bus at home and abroad are using redundancy technology, network structure optimization and anti electromagnetic interference circuit design[2,3]. But in the traditional redundancy technology, fault detection and judgment of CAN bus system are prone to failure, and the reliability of the system is not greatly improved on the whole. Considering the limitation of the traditional redundant technology, a kind of redundant communication system with high reliability is designed from the design of redundant nodes and the way of bus redundancy.

\section{Hardware design of redundant system}

The basic form of node redundancy is the drive level redundancy, controller level redundancy and system level redundancy. The system level redundancy is the most reliable, but the software design and the circuit structure is complex, and the reliability of drive level redundancy is the lowest [4]. The redundant nodes in the controller level are used in this paper. With two enhanced CAN (eCAN) module F2808DSP chip as a dual redundant CAN system processor, and using two eCAN modules inside the F2808 chip, the control node based on dual redundancy CAN communication interface can be formed[5,6].

This system is a universal CAN bus data communication system with DSP as its core, which belongs to a minimum system node of CAN bus. It has the characteristics of generality, practicability and expansibility in the case of stable performance which ensured by structure designing. The CAN bus communication system is mainly composed of microcontroller DSP and CAN bus transceiver, and its communication structure is shown in Figure 1. 


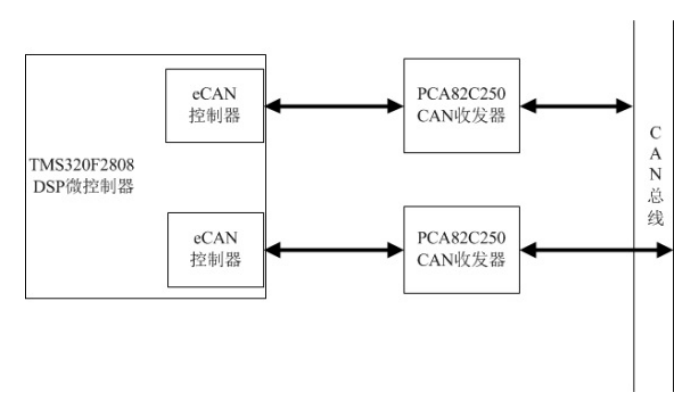

Fig. 1 Redundant CAN bus structure

TMS320F2808 chip is integrated with the enhanced CAN controller (eCAN), which can be easily accessed to the CAN bus system. Therefore, this module design only need to connect the CAN bus driver in the outside without adding the CAN controller to realize the bottom layer of the CAN bus protocol, which has a good expansibility. The hardware circuit of CAN bus interface is shown in Figure 2.

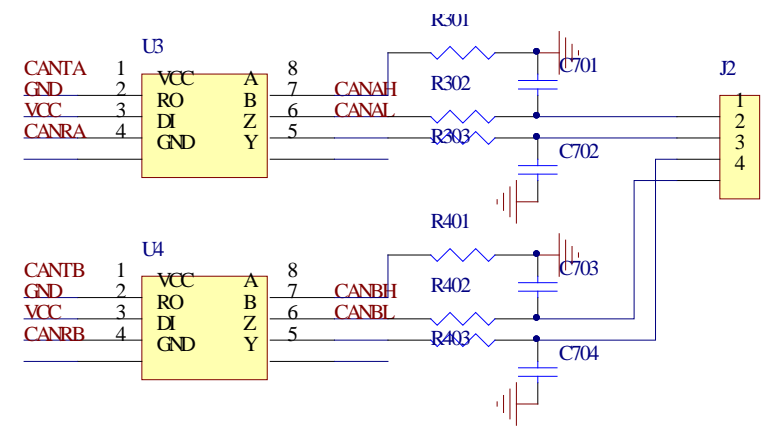

Fig. 2 The hardware circuit of CAN bus interface

Here, CANTA, CANRA and CANTB, CANRB (CANTA, CANRA, CANTB and CANRB are the CAN pins of the DSP chip 2808) are correspondingly connected to the CAN pin on the driver chip U3, U4; J2 is a dual redundant CAN interface, which is connected to the corresponding external CAN bus and external node to realize communication function.

\section{Software design of redundant system}

\subsection{System initialization}

Before sending the information, the system should be initialized, including the initialization of the system controller and the initialization of the eCAN module. The system configuration of the controller is divided into PLLCR register assignment to get the desired system clock frequency, assign high and low speed peripheral clock registers to obtain the desired peripheral clock frequencies and Enable the use of peripheral clocks. The eCAN module initialization process mainly includes the baud rate of configuration, the ID of sending and receiving mailbox, priority and the configuration of data byte length[7,8]. The initialization flow chart is shown in Figure 3. 


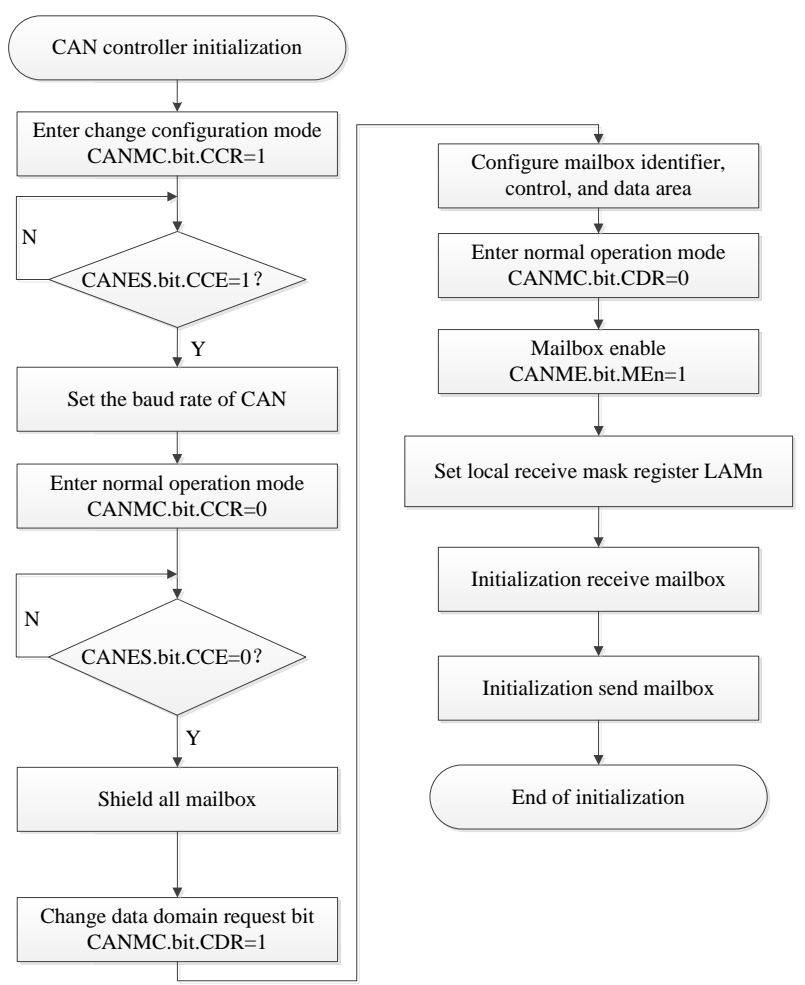

Fig. 3 System initialization flow chart

\subsection{Redundancy management of message}

For dual bus redundant system running simultaneously, the system needs to copy the sending message and send out the system at the same time on the two bus before sending the message[9,10]. When the receiving node receives the message, the first arrived message is firstly received based on the principle of packet priority arrival. After the arrival of the redundant message is not received and directly removed, which ensure that the received message is not repeated. And the reliability of the system is guaranteed in this way. The key of message redundancy management lies in how to realize the efficient filtering of redundant message. The design of redundant message management method is shown in Figure 4. In order to distinguish redundant packets and reduce the complexity of message processing, 8 bit redundant loop serial number are added directly in the 28 bit identifier of the message frame, and the serial number range is 1 255. A message sent to the same node will be cyclic numbered by using the redundant serial number. After sending a message, the redundant serial number plus 1 . When the serial number increases to 255 , the redundant serial number will be restarted from 1 . When the system is reset, the redundant loop number is 0 . The receiving node establishes a redundant serial number in the receive buffer. When the node receives the message, the redundant loop number in the message is extracted, and will be compared with the serial number. If the serial number is the same, the message can not be received. If the serial number is different, the message is received, and the serial number is updated in the serial number. The redundant packets can be efficiently and accurately filtered out with this approach. 


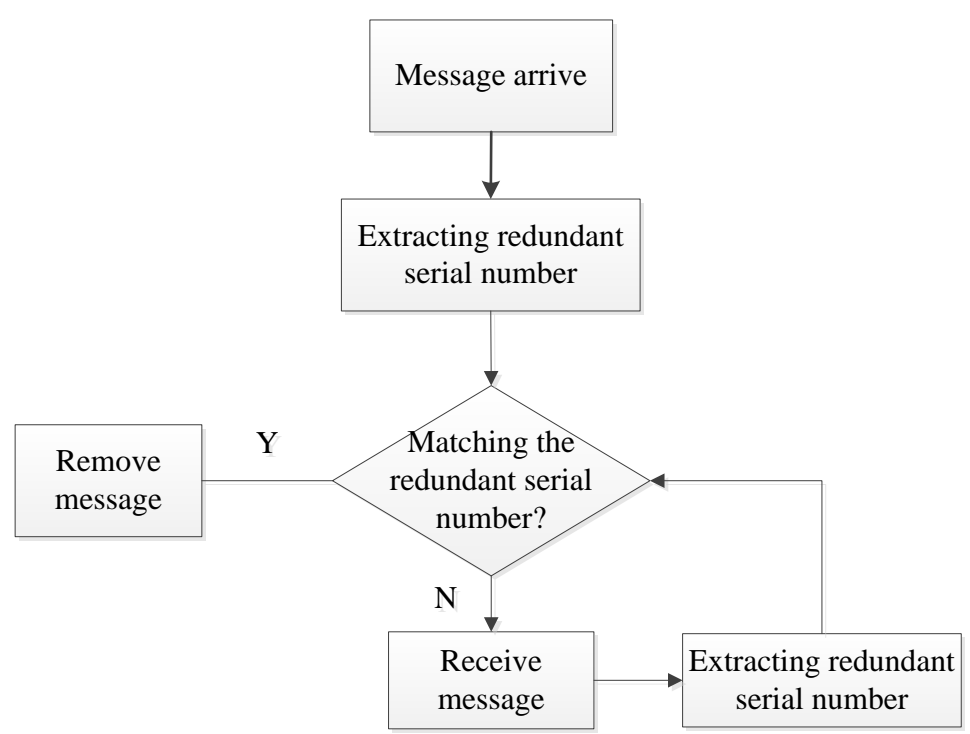

Fig. 4 Redundant management flow chart

\section{System function test}

The design of dual CAN bus redundant communication system is shown in figure 5. The dual CAN interface of DSP2808 redundant nodes are correspondingly connected to the two CAN channels of the ZLG USBCAN-II, and the ZLG USBCAN-II is connected with computer. During the test, redundant CAN nodes send data to the computer, and the computer receives information through the ZLG CANTest software.

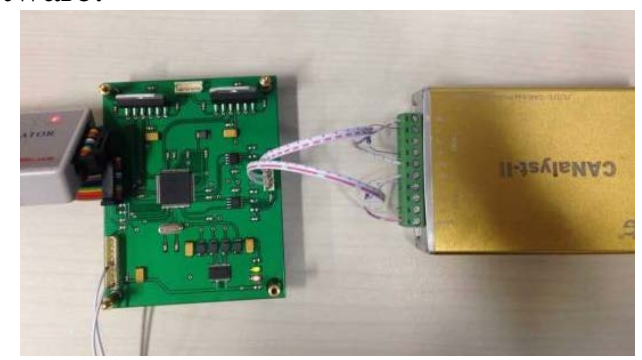

Fig. 5 System communication test

The experimental results show that the dual CAN redundant system which dual bus working together can transmit message well under the dual bus redundancy method. And if one bus failure, the system can still send data through the other bus. And the fault bus has no effect on the normal bus in data transmitting. Therefore, the redundant system has high reliability.

\section{Summary}

A dual CAN redundant communication system based on DSP F2808 chip is designed in this paper. The eCAN controller with built-in F2808 microcontroller is fully utilized in the design of hardware structure, which simplifies the circuit design, reduces the system cost, improves the system integration and reliability; At the same time, the communication system adopts bus redundancy mode of double bus operation, which overcomes the influence of the delay and the reliability of system failure identification, and reduces the recognition time. Experimental results show that the redundant nodes can be used for data transmission of dual channel and single channel fault conditions, which greatly improve the reliability of communication system. However, it can be further optimized and improved in the way of processing redundant message.

\section{References}

[1] Jiang X Y, Zhao K. Comparison of several military vehicle data buses [J]. Ordnance Industry Automation, 2005,05:33-34. 
[2] Chen Y, Sun H X, Jia Q X, et al. Development of the space robot hot and cold dual redundant CAN bus system [J]. Electronic Technology Application, 2008,02:83-86.

[3] Gao J J, Yu Y, Zheng Q W, et al. Dual redundancy network design for weapon system high speed and high reliability application[A]. China command and control society. Proceedings of the third China command and Control Conference (on) [C]. China command and Control Institute: 2015,5.

[4] Yu C L, Xu H L, Liu G W, et al. Research of the Redundant Methods of CAN [J]. Measurement and Control Technology, 2003,10:28-41.

[5] Huang L. Research on the reliability of CAN bus based on Redundancy Technology [D]. Wuhan University of Technology, 2014.

[6] TEXAS INSTRUMENT. TMS320x28xx, 28xxx DSP Enhanced Controller Area Network (eCAN) Reference Guide. USA: TI, 2006. 11-30.

[7] Ren X F. Design of dual CAN bus communication interface circuit based on DSP [J]. Automation and Instrumentation, 2015,08:108-109.

[8] Su H P, Shen C, Liu W Y, et al. Design of real time communication system based on redundant CAN bus [J]. Automation and Instrumentation, 2013,01:26-29.

[9] Wang H L, Tian S S. Dual redundancy method of CAN bus network based on node redundancy [J]. Journal of Detection and Control, 2010,04:90-94.

[10] Wang M Y, Gao M Y. Dual CAN redundant design based on STM32F105 micro controller [J]. Journal of Hangzhou Dianzi University, 2011,02:9-12. 